\title{
Membres en confinement
}

Marianne Roth

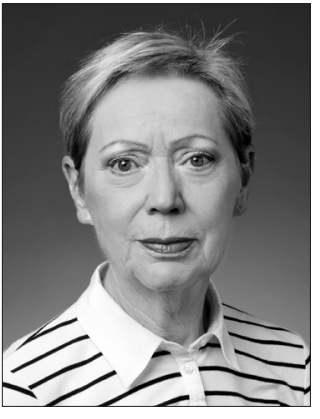

Instantané

La pandémie de COVID-19 a déclenché chez beaucoup de nos membres une grande agitation et beaucoup d'inquiétudes. Même si nous avons essayé de vous transmettre les décisions en cours du Conseil fédéral avec le plus de précision possible, des questions et des incertitudes sont restées sans réponse concernant les effets que celle-ci aura pour la pratique future. Pour nous en tant quassociation professionnelle, il était important d'apprendre de la part de nos membres où le bât blessait et comment nous pouvions apporter notre soutien, ce qui nous a amené à procéder à une brève enquête auprès de la base de nos membres. ${ }^{1}$

\section{Incertitude générale}

Le fait que l'Office fédéral de santé publique (OFSP) n'ait cessé, par manque d'expérience avec le virus, d'avoir des difficultés à donner des instructions compréhensibles, a suscité chez les membres de grandes incertitudes, et de temps en temps aussi beaucoup d'énervement. L'« Ordonnance $\mathrm{N}^{\circ} 2$ relative aux mesures de lutte contre le coronavirus (COVID-19)» a commencé par prescrire en mars que les psychothérapeutes devaient, pendant les deux mois à venir, se contenter de traiter les seuls cas d'urgence, mais que les cabinets devaient rester ouverts. Cette mesure a en même temps automatiquement exclu que les psychothérapeutes installés à leur compte puissent bénéficier de l'allocation au titre de l'indemnité journalière, qui avait été généreusement versée au cours de la période de pandémie de COVID-19. Comme toute une série de travailleurs indépendants, notre profession avait tout simplement été manifestement oubliée par cet organisme de prévoyance, ce qui a plongé beaucoup de nos membres dans une crise existentielle. En intervenant en unissant nos forces et à tous les niveaux politiques, nous avons finalement obtenu que même les psychothérapeutes

1 Lenquête anonyme sur Internet réalisée auprès des membres a eu lieu les 4 et 5 mai 2020 dans les trois régions linguistiques et 280 membres y ont répondu dans l'espace d'une semaine. Outre le fait de répondre à quatre questions concrètes, les membres ont été invités à faire des commentaires supplémentaires sur leur situation. installés à leur compte puissent bénéficier de l'allocation au titre de l'indemnité journalière.

De nombreux membres étaient en outre préoccupés par la question de savoir si leurs patientes et patients, et leurs clientes et clients qui avaient soudain interrompu leurs thérapies pour diverses raisons reviendraient. De nombreuses personnes interrogées étaient d'avis que les véritables effets n'apparaîtraient que plus tard, et se demandaient ce que cela pouvait bien signifier pour leur propre avenir. Certains membres étaient également déstabilisés, parce qu'ils appartenaient eux-mêmes à un groupe à risque, et ne savaient s'ils pouvaient travailler.

\section{Discrimination de la psychothérapie déléguée par rapport à la psychiatrie}

La principale source d'énervement était d'ignorer si les consultations téléphoniques pour les psychothérapeutes à leur compte seraient rémunérées ou non, du fait que ceci nétait réglementé ni au niveau cantonal, ni au niveau fédéral. Nous n’avons même pas reçu de réponses à des demandes réitérées, si bien que nous continuons à rester aujourd'hui encore dans l'incertitude.

Il en a été tout autrement de la psychothérapie déléguée qui est réalisée sous la responsabilité d'une ou d'un médecin et dans le cabinet de cette dernière ou ce dernier. Les consultations téléphoniques étaient certes autorisées et tarifées. Mais lorsque l'OFSP a publié l'instruction en vertu de laquelle les consultations téléphoniques pendant la pandémie passeraient pendant six mois de 240 à 360 minutes, cette annonce n'a pu tout au plus que susciter l'incompréhension. Cette disposition a fait lobjet de critiques unanimes, non seulement de la part des psychothérapeutes, mais aussi de celle des médecins. Elle serait particulièrement choquante, sachant pourtant que de nombreuses patientes et patients, et leurs clientes et clients ne voulaient pas quitter leurs appartements ou utiliser des moyens de transport publics de peur dêtre contaminés. Par opposition, les psychiatres se sont vus accorder pratiquement un temps illimité pour des psychothérapies téléphoniques, ce qui a suscité de grandes incompréhensions et beaucoup de colère, et était tout simplement déconcertant.

Notre demande émise auprès de l'OFSP de supprimer cette injustice a reçu la réponse lapidaire 
que l'extension à 360 minutes correspondrait bien à une augmentation. Le fait que ceci corresponde tout juste à une heure téléphonique de consultation par mois nous a laissés sans voix.

\section{« Restez chez vous »}

Lappel «Restez chez vous » et la fermeture des écoles a placé les parents ayant des enfants devant des défis particulièrement importants. Le cas des familles confrontées à cette situation inhabituelle a souvent été décrit. Deux aspects ont été particulièrement mis en avant au cours de notre enquête. Notamment les psychothérapeutes qui sont des mères élevant leurs enfants seules, travaillaient chez elles et devaient en outre gérer les devoirs scolaires de leurs enfants, ont décrit cette situation comme une expérience limite, tant pour elles-mêmes que pour leurs enfants.

Le deuxième aspect concerne le traitement effectif d’enfants par des thérapies, ce qui a été décrit comme très difficile voire impossible dans des conditions de restrictions. Il serait par exemple impossible de désinfecter des jouets ou de porter un masque, particulièrement au contact de petits enfants. Les thérapies avec des enfants auraient de ce fait pris du retard ou ont dû être carrément annulées.

Comme nous n'avons pas cessé de souligner qu'il fallait autant que possible éviter d'interrompre les thérapies, nous avons recommandé dans notre concept de protection que les enfants amènent leurs propres jouets en thérapie, et d'utiliser une protection contre les crachats en fonction des situations.

\section{Âge de la retraite et groupes à risque}

Certains membres ayant atteint lâge de la retraite ont semblé prendre le prétexte de la pandémie de COVID-19 pour fermer leur cabinet, à moins qu'ils ne l'aient de toute façon prévu depuis longtemps, la crise ayant fourni l'incitation bienvenue pour le faire.

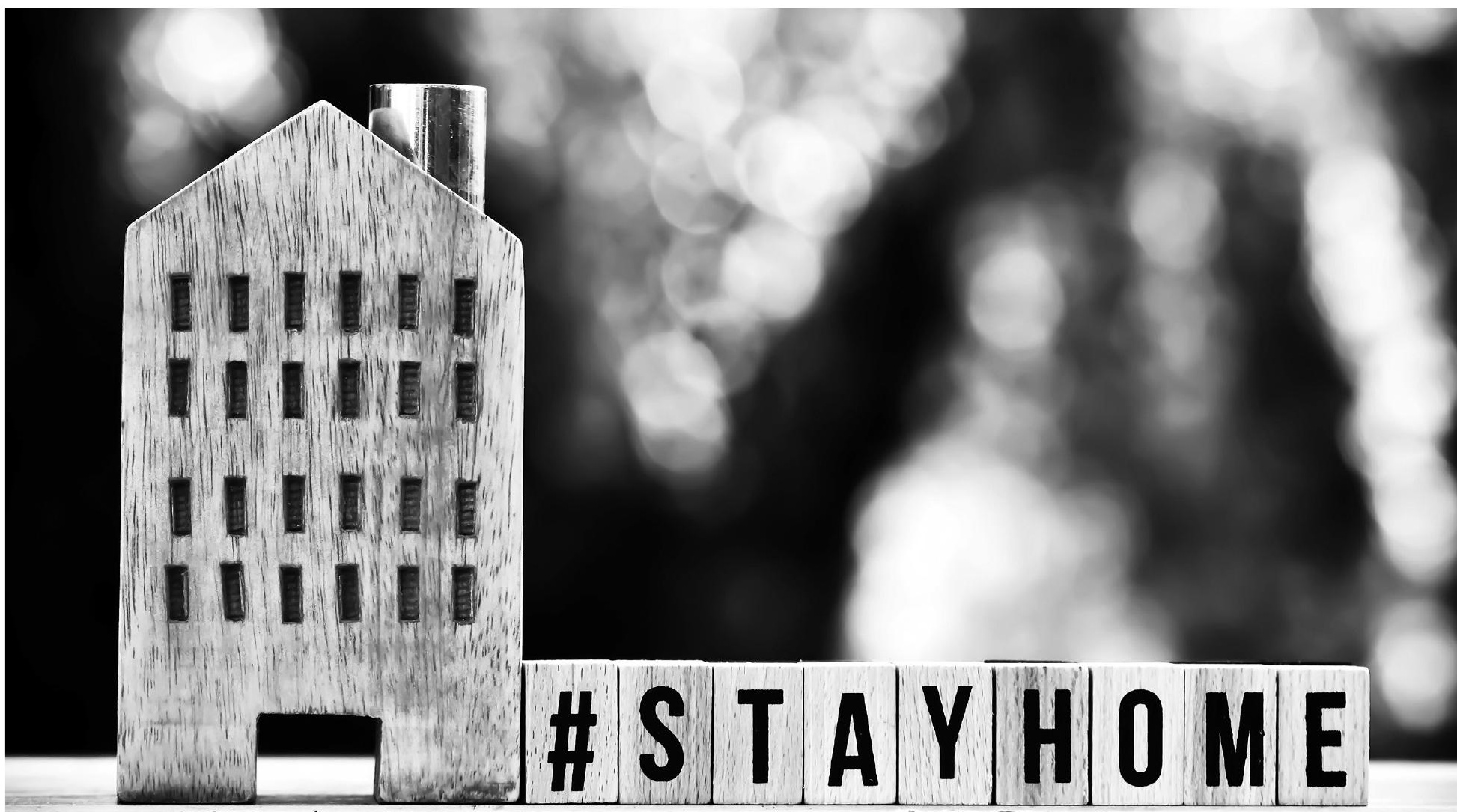


Concernant la situation économique de ce groupe, les personnes interrogées ont exprimé des opinions très variées. Léventail des réactions allait de membres ayant de sérieuses peurs existentielles, du fait de leur appartenance à un groupe à risque ayant dû supporter de grandes pertes financières, jusquà des déclarations de se sentir privilégié à lâge de la retraite. Largent de la caisse de pension et l'AVS permettraient en effet d'affronter les temps avec sérénité.

Avec l'accès à l'allocation au titre de l'indemnité journalière aux travailleurs indépendants, nous avons pu obtenir que même les psychothérapeutes ayant atteint lâge de la retraite puissent faire valoir leurs droits à percevoir des prestations. La condition préalable était qu'ils continuent à verser les cotisations AVS dans la prévoyance vieillesse au-delà de lâge de la retraite et disposent d'un revenu qui était néanmoins limité vers le bas et vers le haut.

Un grand nombre de membres ont rapporté qu'ils traitaient des patients appartenant eux-mêmes au groupe à risque et ne pouvaient ni ne voulaient quitter leur logis. Le téléphone et le chat par vidéo ont fourni ici une alternative bienvenue.

\section{Utilisation d'outils en ligne et thérapie téléphonique}

Pendant que certaines personnes interrogées ont ressenti les thérapies téléphoniques ou les chats vidéo comme une bénédiction, dautres les ont strictement rejetés, sachant que les pour et les contre séquilibraient à peu près. Les partisans étaient unanimes pour dire que la thérapie en ligne serait justement une nécessité de nos jours. Il y avait même des patients qui réclamaient des thérapies téléphoniques, tandis que d'autres au contraire les rejetaient strictement.

Du côté des psychothérapeutes, on sentait bien que des membres aimant utiliser les ordinateurs navaient aucun problème à opérer ce basculement. C'est également ce groupe qui a exprimé le besoin de continuer à pratiquer les thérapies téléphoniques après la pandémie. Lautre groupe trouvait que cela ne convenait pas, et qu'il était éprouvant de ne pas avoir son interlocuteur physiquement en face de soi. C'est également ce groupe qui est le plus confronté à des peurs existentielles. On ne peut pas en déduire une limite d'âge.

Il y a eu des réactions en vertu desquelles les consultations téléphoniques se seraient avérées d'un grand secours, et seraient la seule voie judicieuse dans la présente situation. Il a par ailleurs été regretté qu'il n'y ait aucune garantie que les caisses d'assurance maladie prennent de telles consultations en charge. Il y a même eu des membres qui ne facturaient pas les entretiens téléphoniques. L'expérience a cependant montré que de nombreuses caisses d'assurance maladie avaient trouvé une manière souple de gérer les thérapies téléphoniques.

Pour nous en tant quassociation, cela veut dire que nous voulons réfléchir sur la manière dont nous voulons promouvoir ce développement et pouvons fournir un soutien à nos membres dans le domaine des thérapies en ligne.

Marianne Roth est directrice générale de l'ASP. 